\title{
Spectral Anomalies of Polychromatic DHGB and Its Applications in FSO
}

\author{
Bharat Kumar Yadav, Member, IEEE, and Hem Chandra Kandpal
}

\begin{abstract}
This paper contains some interesting experimental results of the study carried out on anomalous spectral behavior due to coherence and polarization change on polychromatic focused dark hollow Gaussian beam (DHGB). On the basis of experimental and numerical analysis, the possibility and significance of DHGB based free-space optical (FSO) links for indoor and outdoor optical communications are explored.
\end{abstract}

Index Terms-Dark hollow Gaussian beam, free-space optical communication, spectral switching.

\section{INTRODUCTION}

$\mathbf{I}$ $\mathrm{N}$ the last few years optical beams with zero central intensity termed as dark hollow beams (DHBs) have been widely investigated due to their numerous applications in atom trapping [1]-[3] and also in demanding research areas like quantum information processing etc.

Apart from these applications, DHBs might also find potential applications in the field of free-space optical communications [4], [5]. The unique structure of DHBs may be ideal to form FSO links. These links may be exploited in wireless optical communications [6] and to design FSO interconnects [7]. Recently, the effect of atmospheric turbulence on various types of DHBs has been analyzed [4]. These investigations reveal that DHBs may be more stable in the formation of FSO links than normal laser beams. The robustness to atmospheric turbulence compared to normal laser beam with at the expense of beam spreading makes such optical beams more significant for far field optical communications.

Broadly we may categorize the DHBs in two groups namely coherent (e.g., laser-induced) and partially coherent (e.g., broadband light induced) dark hollow beams. Both types of DHBs have some advantages and some limitations. Recently, an advanced technique to produce high quality partially coherent DHB has been demonstrated and it has been emphasized that such beams are having advantages over the coherent beams [8].

Generally, laser is used in FSO communications. Thus, laser-induced DHBs have always been attractive for researchers. The laser is good means for FSO communications

Manuscript received September 23, 2010; revised January 25, 2011; accepted January 25, 2011. Date of publication February 04, 2011; date of current version March 14, 2011.

The authors are with the National Physical Laboratory (Council of Scientific and Industrial Research), New Delhi, India (e-mail: byadav@ mail.nplindia. ernet.in; hckandpal@mail.nplindia.ernet.in).

Color versions of one or more of the figures in this paper are available online at http://ieeexplore.ieee.org.

Digital Object Identifier 10.1109/JLT.2011.2110635 but not for the eye safety [9]. It is assumed that the polychromatic light induced DHB (PLI-DHB) might provide ultimate solution for FSO communications.

Despite their great potential, the polychromatic light induced FSO links [10] have not so far attracted much attention due to their experimental limitations. However, recently, spectral anomalies of PLI-DHB have been reported [11]. Such optical beam may be exploited to form PLI-DHB based FSO links and spectral anomalies (more precisely spectral switching) around critical direction may be used to exchange information in free-space [10]. The spectral switching could be controlled by changing coherence [11] or polarization [12]. These links may be utilized for indoor as well as outdoor optical communications.

In this paper, we present the results of our recent study on partially coherent DHGB produced using polychromatic light. The study includes the generation of DHGB using lens aperture system, and the effect of coherence and polarization on polychromatic light induced DHGB (PLI-DHGB). A separate section is dedicated to explore the possibilities to form and control DHGB based FSO links.

\section{THEORETICAL BACKGROUND}

Suppose a spatially coherent polychromatic hollow Gaussian beam is incident on an aperture lens with full width $2 a$ and focal length $f$, the spectrum density of the field at the observation point $(\vec{r}, z)$ can be expressed as [11]

$$
\begin{aligned}
S(\vec{r}, z ; \omega)= & \frac{S^{(0)}(\omega) Z_{R}^{2}(n !)^{2}}{z^{2}}\left(\frac{\omega}{\omega_{0}}\right)^{2} \\
& \times\left|\sum_{m=1}^{1} \frac{A_{m}}{p^{n+1}} \exp \left[-\frac{Z_{R}^{2} r^{2} \omega^{2}}{z^{2} p w_{0}^{2} \omega_{0}^{2}}\right] L_{n}\left[\frac{Z_{R}^{2} r^{2} \omega^{2}}{z^{2} p w_{0}^{2} \omega_{0}^{2}}\right]\right|^{2}
\end{aligned}
$$

where

$$
\begin{aligned}
& p=1+\frac{B_{m}}{\delta^{2}}+i \frac{Z_{R}}{z} \frac{\omega}{\omega_{0}}-i Z_{F} \frac{\omega}{\omega_{0}} \\
& p=\frac{a}{w_{0}}
\end{aligned}
$$

The terms $Z_{R}=\left(k_{0} w_{0}^{2}\right) /(2)$ and $Z_{F}=\left(k_{0} w_{0}^{2}\right) /(2 f)$ represent the Rayleigh distance and Fresnel numbers respectively while $L_{n}(x)$ denotes Laguerre polynomial of order of $n$. Here, $A_{m}$ and $B_{m}$ are expansion of Gaussian coefficients, $w_{0}$ is beam waist width, $r$ is position coordinate, $z$ is distance between aperture lens and observation point while $k=2 \pi / \lambda$ represents wave 


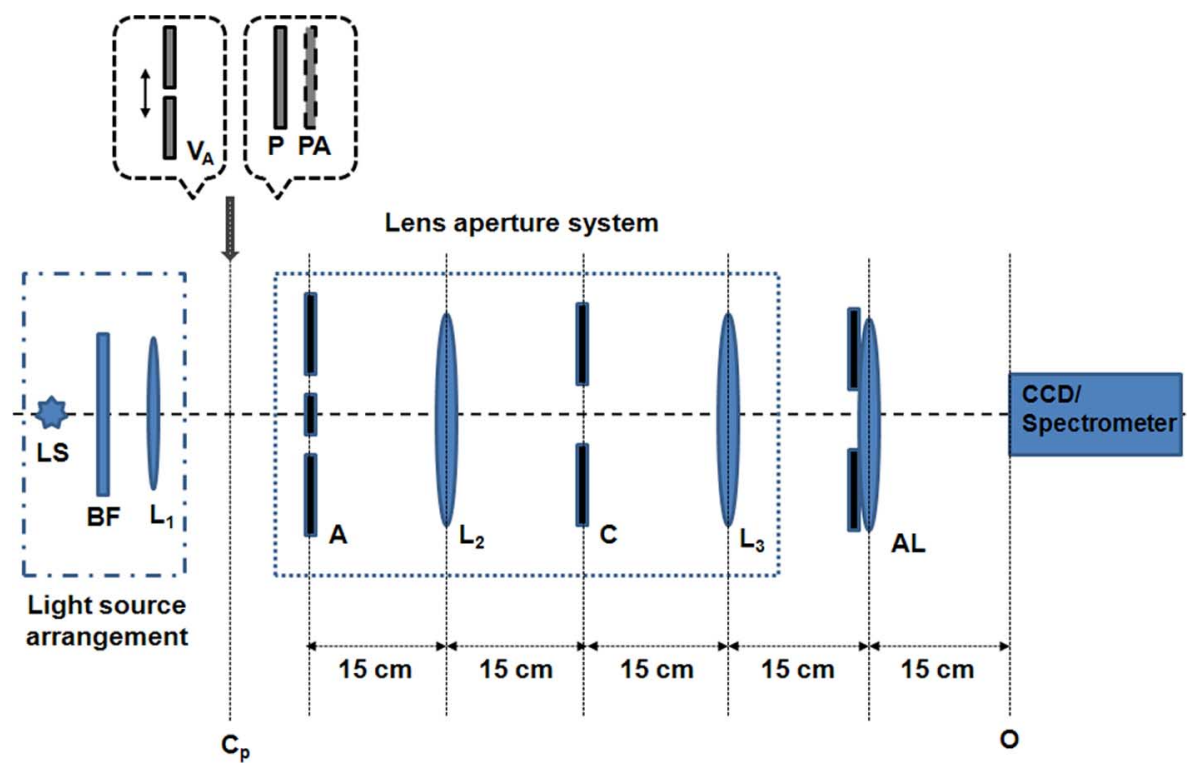

Fig. 1. Schematic of experimental setup.

number. $S^{(0)}(\omega)$ is spectral profile centered at frequency $\omega_{0}$ and bandwidth $\sigma_{0}$ :

$$
S^{(0)}(\omega)=S_{0} \exp \left[-\frac{\left(\omega-\omega_{0}\right)^{2}}{2 \sigma_{0}^{2}}\right]
$$

where $S_{0}$ is a constant.

\section{EXPERIMENTAL INVESTIGATIONS}

A schematic diagram of the experimental setup is shown in Fig. 1. Here LS ( $250 \mathrm{~W}, 24 \mathrm{~V}$ tungsten halogen lamp), BF, A, C and $\mathrm{AL}$ are light source, broadband filter, annular aperture, circular aperture and aperture lens respectively, while $\mathrm{L}_{1}, \mathrm{~L}_{2}, \mathrm{~L}_{3}$ are lenses. The lenses $\mathrm{L}_{2}, \mathrm{~L}_{3}$ and lens with $\mathrm{AL}$ are having same focal length, i.e., $15 \mathrm{~cm}$. The lens aperture system (set of A, $\mathrm{L}_{2}, \mathrm{C}$ and $\mathrm{L}_{3}$ ) is indicated by a dotted line rectangle in Fig. 1 while a dashed dotted line rectangle depicts the light source arrangement. At the back focal length of lens $\mathrm{L}_{3}$ we used CCD to record 3-D images of the DHGBs. A spectrometer was placed to record the spectral profiles of the DHGBs. In Fig. 1, $\mathrm{C}_{\mathrm{P}}$ indicates a plane where we placed variable aperture, $V_{A}$ (to change coherence very precisely) or polarizer, $\mathrm{P}$ and analyzer PA (to change the degree of polarization). $\mathrm{O}$ denotes the observation plane. The experimental investigations were carried out in two parts.

\section{A. Coherence Effect on Spectral Anomalies in DHGB}

After proper alignment of the optical components in the experimental setup (see Fig. 1), a CCD was placed at the back focal plane of AL. A special aperture (or variable aperture, $V_{A}$ ) whose radius could be changed very precisely was introduced between lens, $\mathrm{L}_{1}$ and annular aperture, $\mathrm{A}$. The 3-D image of the PLI-DHGB is shown in Fig. 2(a). To record the spectral profile of this optical beam, the CCD was replaced by a spectrometer. The spectral profile (two-equal-peak spectrum) is depicted in Fig. 2(b). The experimentally observed spectral profile is illustrated by solid dots with uncertainty bars. Each dot denotes an

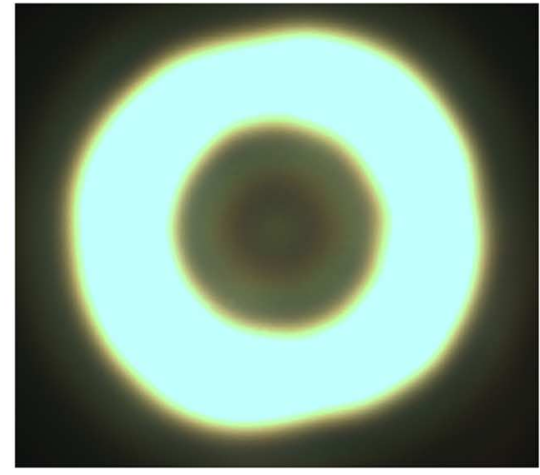

(a)

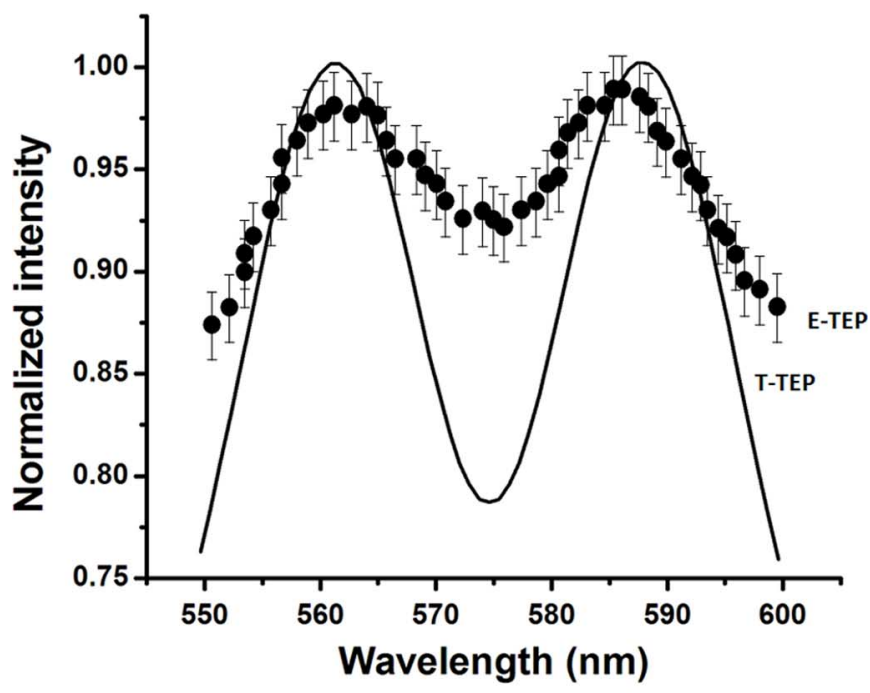

(b)

Fig. 2. DHGB and its spectral profile.

average value of 10 observations. Theoretically expected curve [from (1)] for the experimental parameters, $r / w_{0}=1.713$ is also shown in Fig. 2(b) by solid line. 


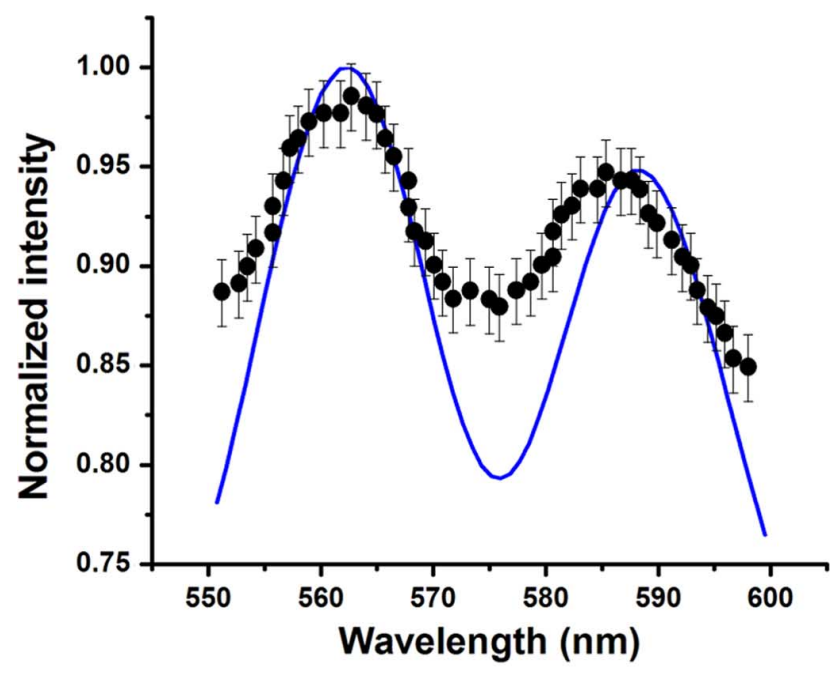

(a)

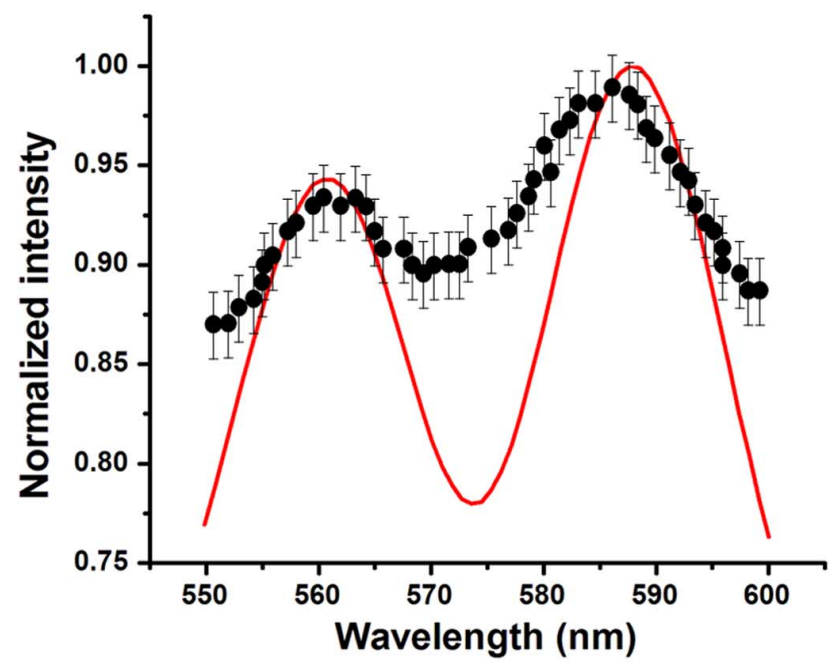

(b)

Fig. 3. Blueshift and redshift in PLI-DHGB.

It is evident from the Fig. 2(b) that the two peaks of experimental curve (E-TEP) are approximately coinciding with the theoretical curve (T-TEP) but the central part of this two-equal-peak spectrum is rather shallow. It is due to the experimental limitations. In our experiment, the resolution of the spectrometer was one of the major factors. To change coherence, the radius of variable aperture, $\mathrm{V}_{\mathrm{A}}$ was changed very precisely. It found that for a particular value of coherence, the resultant spectrum either shifts towards lower wavelengths (blueshift) or higher wavelengths (redshift) with respect to the source spectrum which has Gaussian spectral profile [the curve is not shown in Fig. 2(b)]. The experimental and theoretically expected curves are shown in Fig. 3. The blueshift [Fig. 3(a)] and redshift [Fig. 3(b)] occur for $r / w_{0}=1.710$ and $r / w_{0}=1.715$ respectively. Here again the solid dots represent the experimental data points while solid line shows the theoretical curves. This indicates that the spectral switching phenomenon is taking place in the phase singularity region of the DHGB and is consistent with the theoretical predictions of [11].

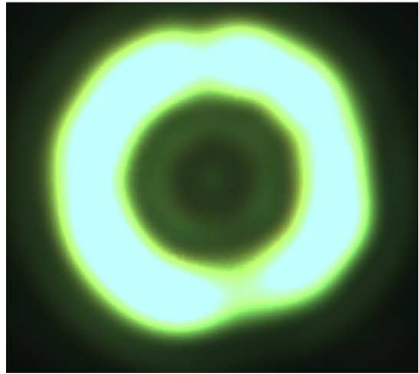

(a)

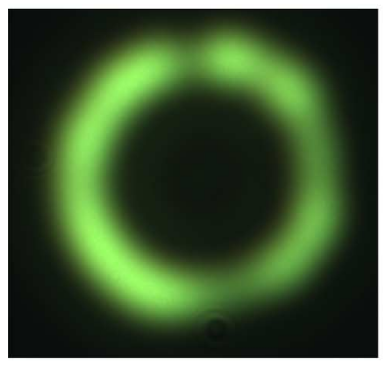

(c)

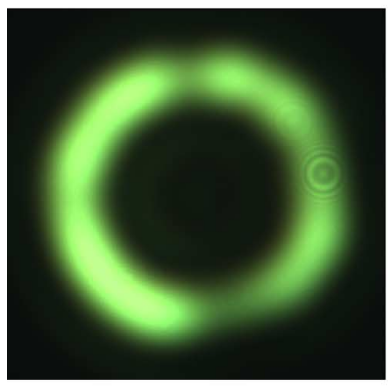

(e)

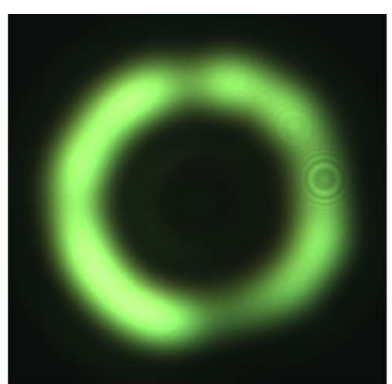

(b)

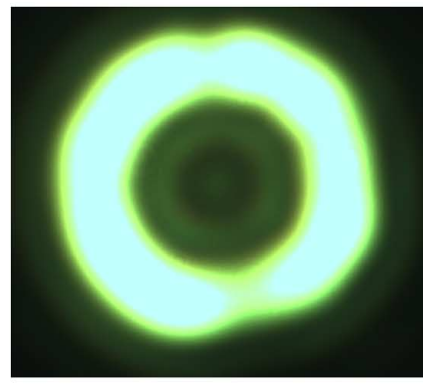

(d)

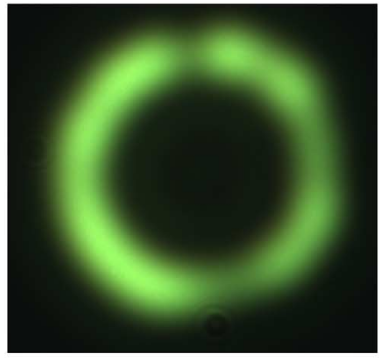

(f)
Fig. 4. 3-D images of DHGB at different polarization angles. (a) $\theta=0^{\circ}$. (b) $\theta=45^{\circ}$. (c) $\theta=135^{\circ}$. (d) $\theta=180^{\circ}$. (e) $\theta=225^{\circ}$. (f) $\theta=315^{\circ}$.

It was observed that the spectral shift is more sensitive towards higher wavelengths in PLI-DHGB optical beam.

Although, the experiment was repeated for two more lenses having focal lengths $f=20 \mathrm{~cm}$ and $f=30 \mathrm{~cm}$ to verify the observations but for the sake of brevity, results obtained with the aperture lens of focal length $f=15$, only are illustrated in Figs. 2 and 3. The theoretical calculations are carried out using (1)-(2) for a range of wavelength from $550 \mathrm{~nm}$ to $600 \mathrm{~nm}$ using $\omega=2 \pi c / \lambda$ and $\omega_{0}=2 \pi c / \lambda_{0}, c$ is speed of light and $\lambda_{0}$ central wavelength. The main experimental parameters are $f=z=$ $15 \mathrm{~cm}, a=1.12 \times 10^{-3} \mathrm{~m}, \lambda_{0}=572.72 \mathrm{~nm}$, and $\sigma_{0}=$ $0.05 \times 10^{15} \mathrm{~s}^{-1}$.

\section{B. Polarization Effect on Spectral Anomalies in DHGB}

Recently, it has been shown theoretically that spectral switches get affected by polarization and more than one spectral switch may be generated by controlling degree of polarization [12]. To analyze the polarization effect on PLI-DHGB, the variable aperture was replaced in the experimental setup (see Fig. 1) by a polarizer, $P$ and an analyzer $P A$ at plane $C_{P}$. This modification reduced the intensity slightly of the resultant DHGB (Fig. 4) with respect to the one obtained with variable aperture, $V_{A}$ [Fig. 2(a)] in the same plane. Fig. 4(a) shows the 


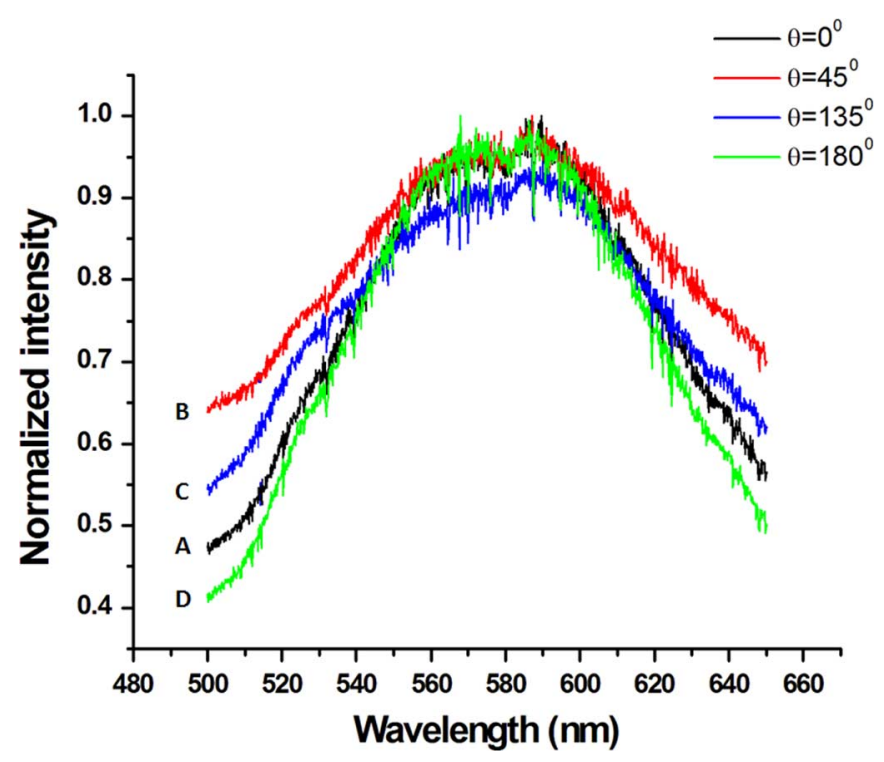

Fig. 5. Spectral profiles of DHGB at different polarization angles.

best possible image for our experimental arrangement. This highest degree of polarization at a particular instant was fixed as a reference where the polarization angle, $\theta=0^{\circ}$.

Further, to observe the polarization effects on the optical beam, we kept on changing the polarization angle by $45^{\circ}$. Some 3-D images of our experimental observations are illustrated in Fig. 4 and some of the corresponding spectral profiles at related polar angles are shown in Fig. 5.

It was observed that at polarization angle $\theta=90^{\circ}$, and $\theta=$ $270^{\circ}$ there was no light while at polarization angles $\theta=0^{\circ}$ [Fig. 4(a)], $\theta=180^{\circ}$ [Fig. 4(d)], and $\theta=360^{\circ}$ (not shown in Fig. 4) we got almost similar 3-D images of the DHGB. The spectral profiles of these DHGBs also show similarities. However, a close examination of the spectral profiles of DHGBs at different polarization angles reveals that despite that the two spectra at $\theta=0^{\circ}$ (curve A in Fig. 5), $\theta=180^{\circ}$ (curve D) overlap at peak level (top part) their tail parts show clear cut separation.

For information encoding and transmission purpose, we are mainly interested in recordable and mutually separable two consecutive spectral switches [two-equal-peak spectrum, see Fig. 2(b)] that take place in a very small proximity of dark region (phase singularity region) in the core of DHGB. Let us take an example where we got this condition. We found that at polarization angles $\theta=135^{\circ}$ (curve $\mathrm{C}$ ) and $\theta=180^{\circ}$ (curve D) the PLI-DHGB shows two spectral switches.

To analyze the separation, we extracted data from these two experimental observations. For the small wavelength range, i.e., $550 \mathrm{~nm}$ to $600 \mathrm{~nm}$, the positions of two spectral switches (curve $\mathrm{C}$ and D) are depicted in Fig. 6. The solid dots are the averaged experimental data for 10 observations while the error bars show the uncertainty region for the corresponding measurements. Here, one can observe clear-cut separation between two consecutive spectral switches. The significance of these spectral switches in optical communication will be discussed in the next section.

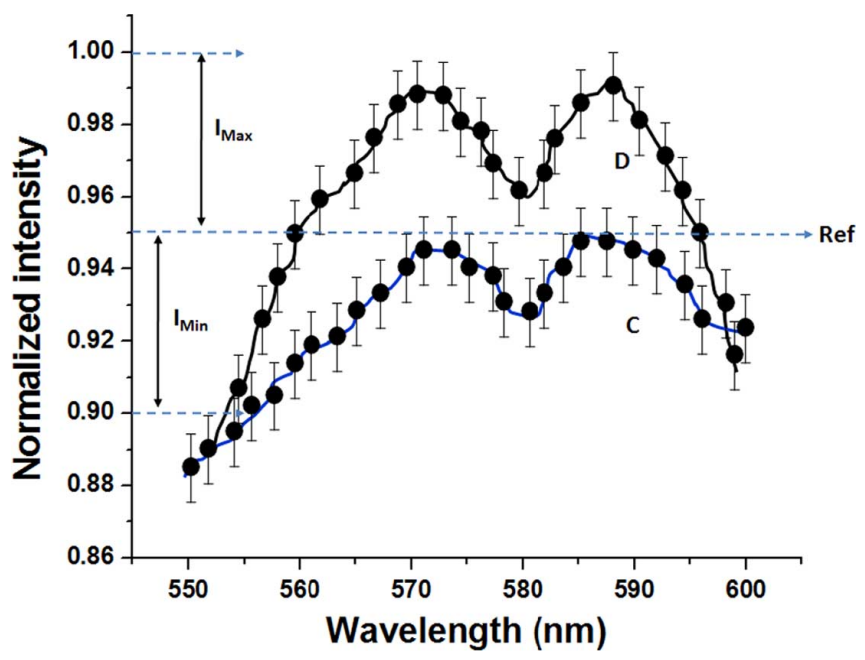

Fig. 6. Two consecutive spectral switches at $\theta=135^{\circ}$ and $\theta=180^{\circ}$.

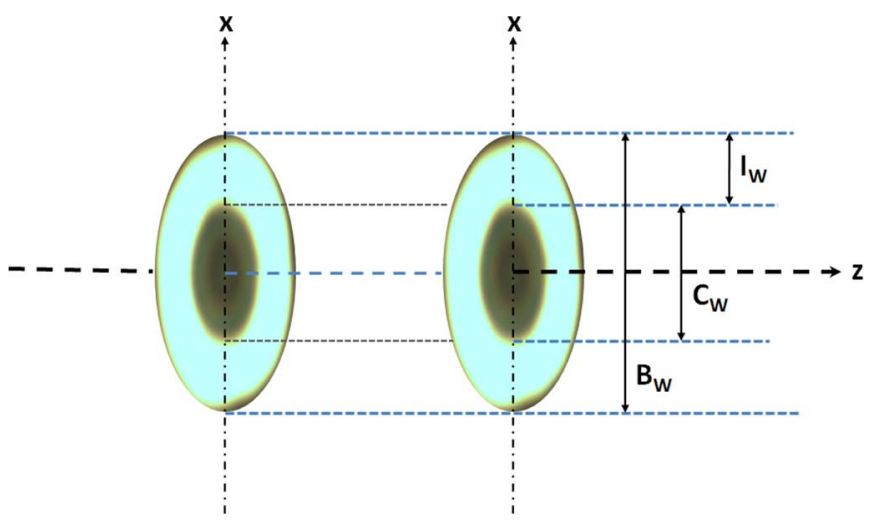

Fig. 7. Virtual optical fiber like structure by DHGB.

\section{DHGB BASED FREE-SPACE OPTICAL LINKS}

Although, the DHGB based FSO link formation is a contrived idea but it has a great potential for realization and might find potential applications in board-to-board, indoor and outdoor optical networks.

\section{A. Origin of the Concept}

As we have mentioned earlier that PLI-DHGB is a special type of optical beam which has phase singularity region (dark hollow) in its core. The dark core of the beam is surrounded by high intensity light. The overall structure of such optical beam appears like a virtual optical fiber. To illustrate the concept, a schematic is shown in Fig. 7. $\mathrm{I}_{\mathrm{w}}, \mathrm{C}_{\mathrm{w}}$ and $\mathrm{B}_{\mathrm{w}}$ respectively indicate width of intensity distribution, dark core of the beam and total width of the PLI-DHGB. During propagation along with z-axis, these widths will vary.

If one establishes the PLI-DHGB based FSO link between two points, the phase singularity region (dark hollow core) may be exploited to process information through spectral switching [10] while the intensity distribution around phase singularity region may behave like protection shield. The over all structure may create a secure and reliable free-space optical link in near field (few centimeters) as well as in far field (few meters or beyond). 


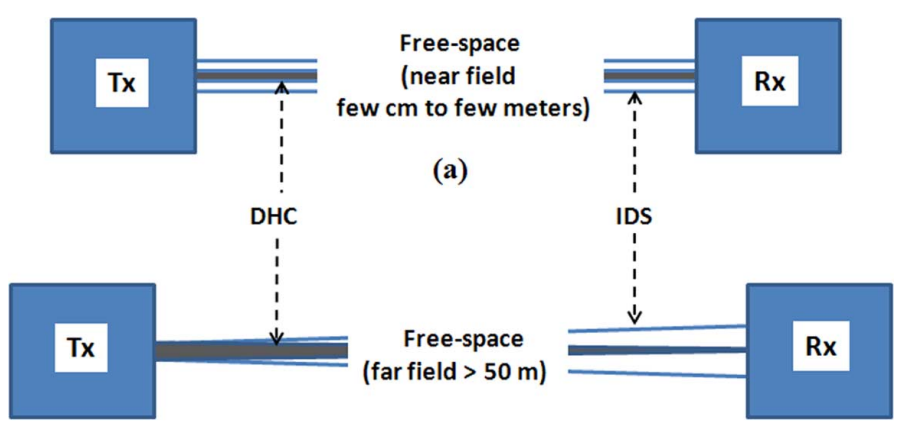

(b)

Fig. 8. Point to point FSO link through PIL-DHGB.

\section{B. Links Formation and Distance Considerations}

These investigations reveal that DHGB shows good propagation stability in the near field but in far field (as we further increase of the propagation distance), the intensity distribution diverges and the dark region across the DHGB decreases. A situation reaches in the far field at which the dark region disappears and the on axis intensity becomes maximal [13]. Hence, the good propagation stability of the PLI-DHGB based FSO links in near zone may be quite suitable for designing free-space optical interconnects. Few meters long links may be exploited for indoor optical links as the beam maintains uniformity in the shape and the size of the virtual fiber link like structure. A typical pictorial example is illustrated in Fig. 8(a).

Theoretically it has been shown [13] that during propagation, at larger distances $(\approx 49.65 \mathrm{~m})$, the dark core of the DHGB may completely disappear. However, this distance depends closely on the Rayleigh distance and the initial coherence of the source beam. It means, at least a maximum distance below $49 \mathrm{~m}$ may be utilized to establish DHGB based FSO links in far field. However, experimentally it is almost impossible to maintain good signal to noise ratio for such a distance using available polychromatic light sources. In our experimental setup the lens aperture system is used to generate DHB at geometrical focal plane of the aperture lens, hence, the distance, $z$ will mainly depend on the focal lengths of the lenses in use. Moreover, one may get good quality optical beam in this type of optical system.

To check the quality of spectral switching in DHGB for larger distances, we performed computer simulation on the experimental parameters using (1). We found that theoretically it is possible to get spectral switching in the system (see Fig. 1) by using lenses of focal lengths $10 \mathrm{~m}$ and $20 \mathrm{~m}$ or beyond but in these cases the optical setup will not be manageable due to increased distances between different optical elements (see Fig. 1). However, for $f=z=10 \mathrm{~m}$, the blueshift, two-equalpeak and redshift occur for $r / w_{0}=41.50, r / w_{0}=41.75$ and $r / w_{0}=41.90$ respectively. Similarly for $f=z=20 \mathrm{~m}$, the blueshift, two-equal-peak and redshift occur for $r / w_{0}=$ $83.2, r / w_{0}=83.51$ and $r / w_{0}=83.67$ respectively. In actual experimental observations, the phase singularity region of the beam sustained up to a few meters beyond the focal plane (depending on the focal length of the lenses) then it started shrinking and finally disappeared resulting in intensity distribution divergence. A hypothetical situation is shown in Fig. 8(b).

\section{Information Encoding Scheme}

One may exploit the spectral switching of polychromatic light for information encoding purpose in three ways namely frequency shift keying (FSK), on-off keying (OOK) and as a function of high variation in consecutive spectral switches.

In FSK scheme with spectral switching, the spectral shifting from lower wavelength (blueshift) to higher wavelength (redshift) or vice-versa with respect to the source spectrum gives spectral flip-flop like condition to encode information [10]. For example, if blueshift could be associated with "1", redshift would automatically be associated with information bit "0" or vice versa. This strategy follows traditional FSK scheme for information encoding. The concept is illustrated in Fig. 9(a) where the source spectrum (Gaussian curve), blueshift, and redshift are shown by different color shades. The solid line on reference spectrum (Gaussian curve) indicates the reference plane where one may get maximum intensity point [MIP, see Fig. 9(a)] at central wavelength while the parallel dashed lines exhibit the planes for corresponding blueshift (left line) and redshift (right line) respectively. Thick arrows show the direction of the spectral shifting.

The OOK like information encoding with spectral switching is also possible. The variation in the central wavelength (sudden drop) due to the phase singularity effect may provide the alternate way to express spectral switching at a fixed point. This concept is shown in Fig. 9(b) where, the MIP at the central wavelength of the source spectrum (Gaussian curve) drops (see thick dotted arrow) in the modified spectrum that results a two-peak spectrum. The intensity flip-flop of MIP at a fixed point (or in a small region) due to phase singularity effect provides another way to encode information. This scheme follows the popular on-off keying (OOK) technique for information encoding. The maximum intensity may be denoted as information bit " 1 " while the central dip of intensity may be associated with "0". According to the requirements any encoding scheme may be used for spectral switching based information processing.

The third information encoding scheme is quite interesting and may be most suitable for information processing through PLI-DHGB. As we have demonstrated earlier, one may get more than one spectral switches in a very small proximity of DHGB. The height of the central dip of the two-equal-peak spectrum may vary in each switch, e.g., curve $\mathrm{C}$ and D in Fig. 6. Let us term the two spectral switches as $\mathrm{I}_{\mathrm{Max}}$ and $\mathrm{I}_{\mathrm{Min}}$ respectively. These switches may be exploited to indicate two different stages for information incoding. The information bits ("1" and "0") may be associated with these stages. A simple example for information encoding scheme with spectral switches is shown in Fig. 9(c). Here $\mathrm{I}_{\mathrm{Max}}$ and $\mathrm{I}_{\mathrm{Min}}$ are associated with information bits " 1 " and "0" respectively. A decimal number "10" which is equivalent to binary value " 1010 " is encoded with the combination of first and second spectral switch, i.e., "I $\mathrm{I}_{\text {Max }} \mathrm{I}_{\text {Min }} \mathrm{I}_{\text {Max }} \mathrm{I}_{\text {Min }}$ ". This scheme is quite similar to the popular OOK information encoding scheme but with different approach. Main advantage of this scheme is smooth information processing using spectral switching through DHGB without intensity fluctuation. 


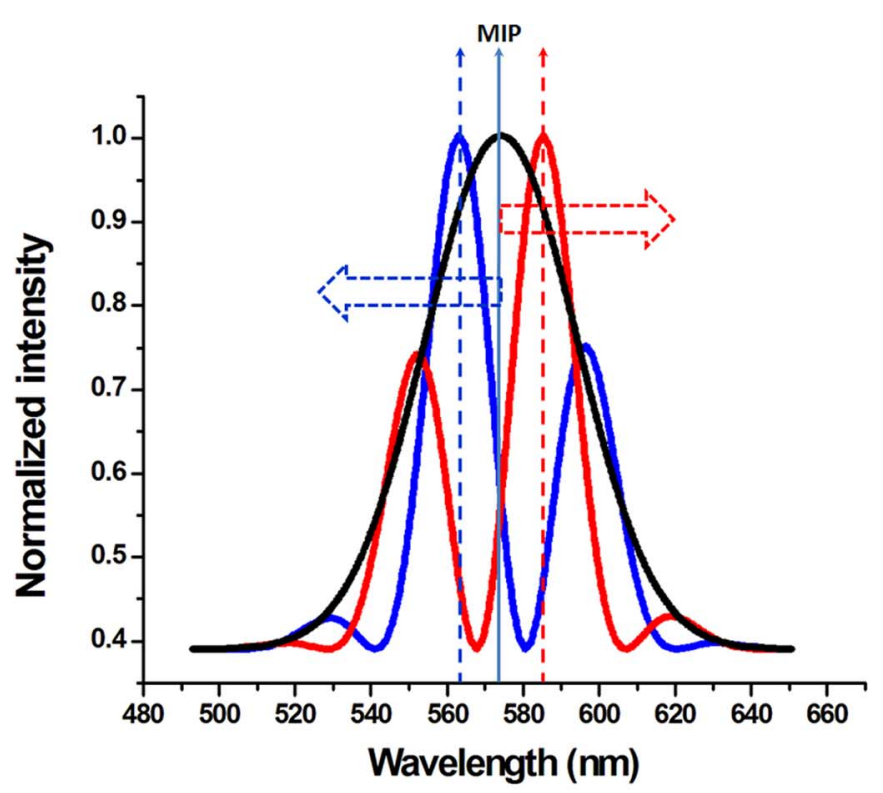

(a)

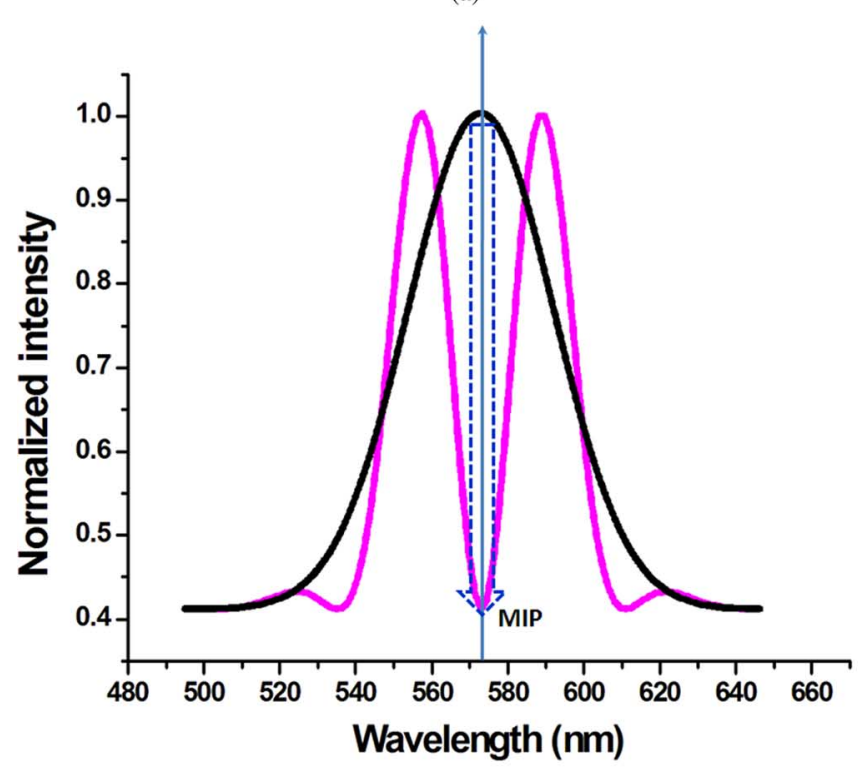

(b)

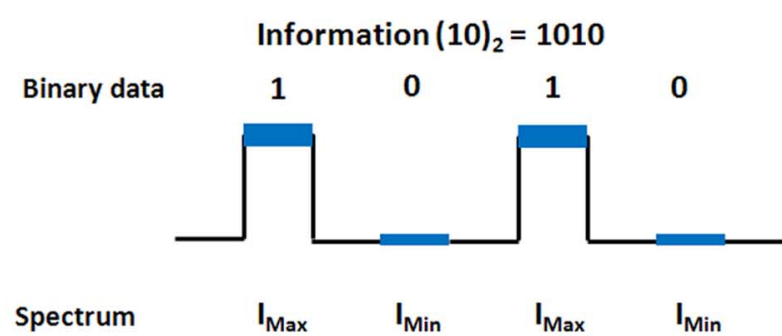

(c)

Fig. 9. Information encoding scheme with spectral switches. (a) FSK. (b) OOK. (c) Information encoding.

The experimental uncertainties may also influence the switch variation. Thus, we may assign intensity fluctuation zone to separate out the two spectral switches. Suppose, if the dip of a switch falls between $>0.95 \%$ to $1.00 \%$ of the normalized intensity, it may be designated as $\mathrm{I}_{\mathrm{Max}}$. Similarly, the intensity range from $0.90 \%$ to $0.95 \%$ may be reserved to designate $\mathrm{I}_{\mathrm{Min}}$ The intensity fluctuation zones are shown in Fig. 6 by arrows while a dashed line indicates the separation boundary of two spectral switches. These are assumptions to explain information encoding scheme within experimental uncertainties.

\section{Spectral Control and Performance}

In the previous section we have demonstrated that spectral switching that takes place in DHGB can be controlled either by coherence change or polarization change. It is found that the spectral switching control by coherence modulation is a cumbersome task. It requires very precise changes and is prone to enhancing experimental uncertainties. Moreover, spectral switching based information processing through this control that supports FSK encoding scheme may provide slow data rate, i.e., Mbsp [10]. Alternatively, the polarization control over spectral switching that supports OOK encoding strategy may give excellent results. If it is realized, one may achieve Gbps level data transmission speed in free-space. It is evident that researchers have already demonstrated ultimate technique to modulate polarization up to $\mathrm{GHz}$ level [14].

\section{IMPORTANCE, GROWTH AVENUES, AND FUTURE CHALLENGES}

The major advantages of the optical communication through DHB based FSO links have ultimate stability and smooth information transmission capability without any intensity fluctuations. Moreover, in recent research, it has already been reported that coherent as well as partially coherent DHBs are less affected by the atmospheric turbulence [15].

Once the optical link is established between two fixed points; the information processing takes place through spectral switching. The spectral switching will be controlled by changing the optical properties of light (coherence or polarization modulation), therefore, no intensity fluctuation interferes the optical communication. On the other hand, in traditional laser based FSO communication using OOK, one can not avoid the significant impact of intensity fluctuations. The problem keeps on increasing as we move towards longer distances. Moreover, PLI-DHGB based FSO links are eye friendly, hence are ideal for indoor optical networks. Despite their possible potential applications in FSO communication, DHBs have some constraints:

- DHB exhibits good propagation stability in near zone but for larger distance, they are not suitable due to their distorting nature during propagation.

- The diffraction and scattering properties of DHGB may also add extra complications to the wireless communication process with such optical beams.

- Polychromatic light induced DHGB has more experimental limitations due to lack of good broadband light source. In near future the so-called white light laser (laser like broad band source) might bring revolution in this field.

- The speed of the overall communication system may highly depend on the modulation speed (i.e., coherence or polarization modulation) and also on the speed of transmitting and receiving ends. 
DHB based FSO link and related technologies seem to be quite significant and promising but the realization of such techniques is not as easy as it sound. It may take a passage of time and requires future technological advancements.

\section{ACKNOWLEDGMENT}

The authors would like to thank the Director of the National Physical Laboratory (CSIR) for his support in this research. They would also like to thank M. Varma for his assistance in experimental work and K. N. Basawaraju for setup arrangement.

\section{REFERENCES}

[1] J. Yin, W. Gao, and Y. Zhu, "Generation of dark hollow beams and their applications," in Progress in Optics, E. Wolf, Ed. Amsterdam, The Netherlands: North Holland, 2003, vol. 44, pp. 119-204.

[2] J. Arlt and M. J. Padgett, "Generation of a beam with a dark focus surrounded by regions of higher intensity: The optical bottle beam," Opt. Lett., vol. 25, pp. 191-193, 2000.

[3] T. Wang, J. Pu, and Z. Chen, "Generation and propagation of partially coherent vortex beams," Optoelectron. Lett., vol. 5, pp. 0077-0080, 2009.

[4] Y. Cai and S. He, "Propagation of various dark hollow beams in a turbulent atmosphere," Opt. Exp., vol. 14, pp. 1353-1367, 2006.

[5] F. E. S. Vetelino and L. C. Andrews, "Annular Gaussian beams in turbulent media," in Proc. SPIE, 2004, vol. 5160, pp. 86-97.

[6] C. C. Davis, I. I. Smolyaninov, and S. D. Milner, "Flexible optical wireless link and networks," IEEE Commun. Mag., vol. 41, pp. 51-57, 2003.

[7] N. Savage, "Linking with light," IEEE Spectrum, vol. 39, pp. 32-36, 2002.

[8] C. Zhao, Y. Cai, F. Wang, X. Lu, and Y. Wang, "Generation of a highquality partially cohetrent dark hollow beam with a multimode fiber," Opt. Lett., vol. 33, pp. 1389-1391, 2008.

[9] S. Qazi, "Challenges in outdoor and indoor optical wireless communications," in Proc. Int. Conf. Wireless Networks, Las Vegas, NV, 2006, pp. 448-458.

[10] B. K. Yadav, S. Raman, and H. C. Kandpal, "Information exchange in free-space using spectral switching of polychromatic light: Possibilities and limitations," J. Opt. Soc. Amer. A, vol. 25, pp. 2952-2959, 2008.

[11] H. Zhang, G. Wu, and H. Guo, "Spectral anomalies of focused hollow Gaussian beams at the geometrical focal plane," Opt. Commun., vol. 281, pp. 4169-4172, 2008.

[12] L. Pan, Z. Zhao, C. Ding, and B. Lu, "Effect of polarization on spectral switches in the diffraction of stochastic electromagnetic beams," Appl. Phys. Lett., vol. 95, pp. 1811121-1811123, 2009.
[13] Y. Cai, X. Lu, and Q. Lin, "Hollow Gaussian beams and their propagation properties," Opt. Lett., vol. 28, pp. 1084-1086, 2003.

[14] J. D. Bull, N. A. F. Jaeger, H. Kato, M. Fairburna, A. Reid, and P. Ghanipour, "40 GHz electro-optic polarization modulator for fiber optic communications systems," in Proc. SPIE, 2004, vol. 5577, pp. 133-143.

[15] Y. Yuan, Y. Cai, J. Qu, H. T. Eyyuboglu, Y. Baykal, and O. Korotkova, "M2-Factor of coherent and partially coherent dark hollow beams propagation in turbylent atmosphere," Opt. Exp., vol. 17, pp. 17344-17356, 2009.

Bharat Kumar Yadav (M'10) was born on June 11, 1971, in Kankroli, Rajasthan, India. He received the B.Sc. degree in science from M. L. Sukhadia University, Udaipur Rajasthan, India, in 1991, the M.C.A. degree from Indra Gandhi National Open University, New Delhi, India, in 2001, and the Ph.D. degree in computer science from Jamia Millia Islamia, New Delhi, India, in 2009.

He is currently a Technical Officer with Optical Radiation Standards, National Physical Laboratory, (Conical of Scientific and Industrial Research), New Delhi, India. He has more than 15 years' work experience in the field of computing, optical measurements, and research. He is currently performing multidisciplinary research work, including computing, optics, and communication. He has been studying the spectral switching phenomenon of polychromatic light and its applications in free-space optical (FSO) and optical computing since 1999. He is the author or coauthor of 21 research articles published in various journals and proceedings of international/national conferences. His current area of interest is dark hollow beams based FSO communication, FSO technologies, information encoding, and information hiding through spectral switching of polychromatic light.

Dr. Yadav is an affiliate member of the IEEE Communication Society.

Hem Chandra Kandpal was born in 1954 in Almora (Uttaranchal), India. He received the B.Sc. degree in science from Agra University, Uttar Pradesh, India, in 1973, and the M.Sc. degree in science and Ph.D. degree from Kumaon University, Nanital, Uttrakhand, India, in 1975 and 1980, respectively.

He has more than 35 years research experience in optical coherence, singular optics, quantum optics, spectroscopy and optical metrology. At present, he is the Head of Optical Radiation Standards and Quantum Optics and Photonics section, National Physical Laboratory, New Delhi, India. He is the author or coauthor of more than 100 research papers for different international and national journals. He has delivered a number of invited talks and has presented numerous papers in international and national conferences. He is actively engaged in finding the applications of optical coherence in optical metrology, and optical computing. His areas of research are optical coherence, singular optics, quantum optics, spectroscopy, and optical metrology.

Dr. Kandpal is a Fellow of the National Academy of Sciences, the Optical Society of India, the Metrology Society of India, and a Life Member of the Laser Society of India. He was the recipient of the CSIR Young Scientist Award in 1990 for his outstanding work on coherence optics. 